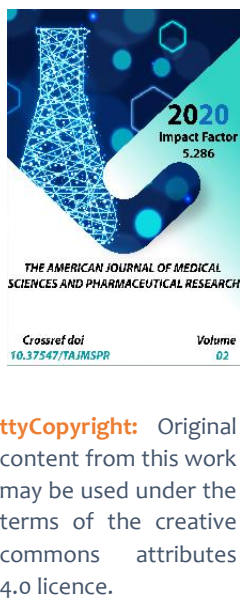
4.0 licence.

\section{Diagnostic Aspects Of Hyperprolactinemia Syndrome}

\section{Z.Y.Khalimova}

D.M.S., Prof. , Department Of Endocrinology And Pediatric Endocrinology, Tashkent Pediatric Medical Institute, Tashkent, Uzbekistan

D.U.Uralova

Master, Department Of Endocrinology And Pediatric Endocrinology, Tashkent Pediatric Medical Institute, Tashkent, Uzbekistan

\section{M.S.Safarova}

RSNPMCE Named After Y.Kh. Turakulov, Tashkent, Uzbekistan

\section{Y.O.Juraeva}

RSNPMCE Named After Y.Kh. Turakulov, Tashkent, Uzbekistan

\section{A.A.Gumarova}

RSNPMCE Named After Y.Kh. Turakulov, Tashkent, Uzbekistan

\title{
ABSTRACT
}

Hyperprolactinemia syndrome is one of the most common neuroendocrine disorders. In the structure of endocrine pathology, it ranks third after diabetes mellitus and thyroid diseases. The article is devoted to the topical issues of diagnosing hyperprolactinemia syndrome depending on the cause of the development of this syndrome.

\section{KEYWORDS}

Prolactin, hyperprolactinemia, hypogonadism, diagnosis, macroprolactinemia, PCOS, prolactinoma, hypothyroidism.

\section{INTRODUCTION}

Hyperprolactinemia syndrome, characterized by excessive pathological secretion of prolactin, accompanied by the development of hypogonadism in men and women, and possibly pathological discharge from the mammary glands, is a very common pathology [1]. In women aged 25-34 years, the incidence of hyperprolactinemia is 23.9 per 100,000 population per year $[2,3]$. The incidence of hyperprolactinemia in women of reproductive 
age is $24.2 \%$ and ranges from 11 to $47 \%$ in various individual studies [4].

Diagnosis of hyperprolactinemia is a difficult task, requiring not only the determination of the content of prolactin (PRL), but also its isoforms with high biological activity, a thorough study of the anamnesis, the exclusion of various somatic, endocrine and neuroendocrine disorders $[5,12,14]$.

\section{OBJECTIVE}

To evaluate the results of laboratory and instrumental research methods in women with hyperprolactinemia of various origins.

\section{MATERIALS AND METHODS}

Examination of patients was carried out on the basis of the Y.Kh. Turakulov in 2018-2020. 90 women with hyperprolactinemia were examined. All examined patients were divided into 3 groups: 30 patients with prolactinomas, 30 patients with polycystic testicles syndrome and 30 patients with hypothyroidism. All patients underwent conventional research methods (general clinical and biochemical blood tests, radioimmunological hormonal blood tests, MRI of the pituitary gland, ultrasound of the pelvic organs). The age of the patients in our study at the time of treatment was $33.5 \pm 11.5$ years, minimum - 17.0 years, maximum - 49.0 years. The duration of the disease ranged from 6 months to 16 years. The age gradation of patients by group is shown in Figure 1.

Diagnosis of hyperprolactinemia syndrome consists of the following stages: laboratory confirmation of hyperprolactinemia, exclusion of symptomatic forms of the disease, exclusion of the phenomenon of macroprolactinemia, visualization of the hypothalamic-pituitary region, visualization of the pelvic organs and assessment of sexual function $[6,7,12]$.

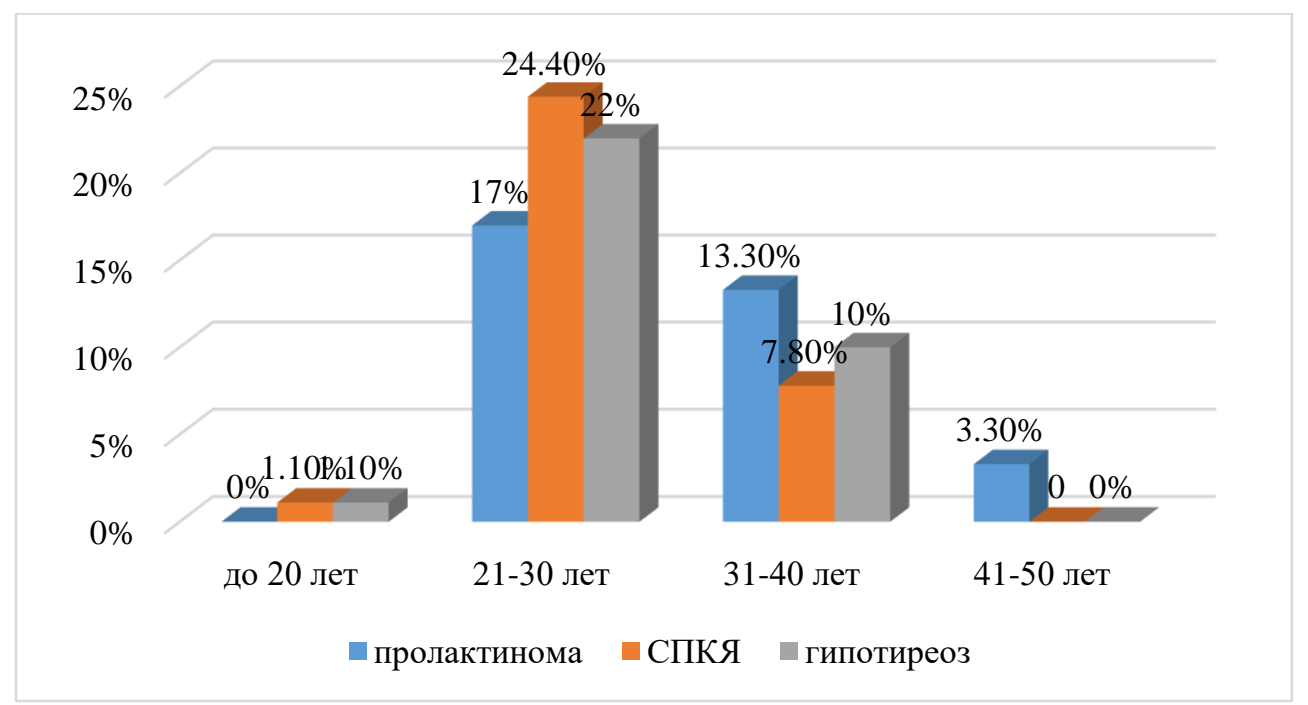

Figure: 1. Age gradation of women with hyperprolactinemia syndrome by groups 
Determination of the basal prolactin level for the diagnosis of hyperprolactinemia syndrome

According to the latest clinical guidelines for the diagnosis and treatment of hyperprolactinemia syndrome released in 2011, to establish the diagnosis of hyperprolactinemia, it is sufficient to determine the level of prolactin in the blood serum once. Currently, from the standpoint of evidence-based medicine, dynamic testing of the prolactin content for the diagnosis of hyperprolactinemia is considered inappropriate [8, 9, 15]. Level RLP above rules confirm diagnosis provided hours the venipuncture made without undue stress to the patient and taking into account all the possible physiological effects on the secretion of prolactin $[10,16,17]$.

According to the literature, with hyperprolactinemia of tumor genesis, the level of prolactin in the blood serum is significantly higher than with hyperprolactinemia of nontumor genesis, since according to international expert recommendations published in 2006, basal prolactin levels can be used to judge the genesis of hyperprolactinemia [11, 18, 19].

The content of the basal level of total prolactin in the total group of examined patients is presented in Table 1.

Table № 1. The level of total prolactin in the examined patients depending on the genesis of hyperprolactinemia

\begin{tabular}{|c|c|c|c|}
\hline & Prolactinoma & PCOS & Hypothyroidism \\
\hline Number of patients & 30 & 30 & 30 \\
\hline Median & 1812 & 963 & 958 \\
\hline Lower quartile & 1224 & 588 & 551 \\
\hline Upper quartile & 2973 & 1488 & 1115 \\
\hline $\begin{array}{c}\text { Kruskal - Wallis } \\
\text { criterion } \mathrm{p}=0.001\end{array}$ & $\mathrm{p}_{1-2}<0.001$ & $\mathrm{p}_{2-3}=0.990$ & $\mathrm{p}_{1-3}<0.001$ \\
\hline
\end{tabular}

An increased level of prolactin is observed in all patients with prolactinoma (30,100\%), in 21 with PCOS (70\%) and in 24 with hypothyroidism (80\%).

When assessing the dependence of the level of total prolactin on the cause of hyperprolactinemia, between tumor hyperprolactinemia (prolactinoma) and non-tumor genesis (PCOS, hypothyroidism), significant differences were obtained (Kruskal - Wallis criterion, $\mathrm{p}_{1-2}<0.001, \mathrm{p}_{1-}$ $3<0.001$ ), but in group of women with hypothyroidism between patients with PCOS, no significant differences were obtained $p_{2-3}=0.990$, Fig. 2. 


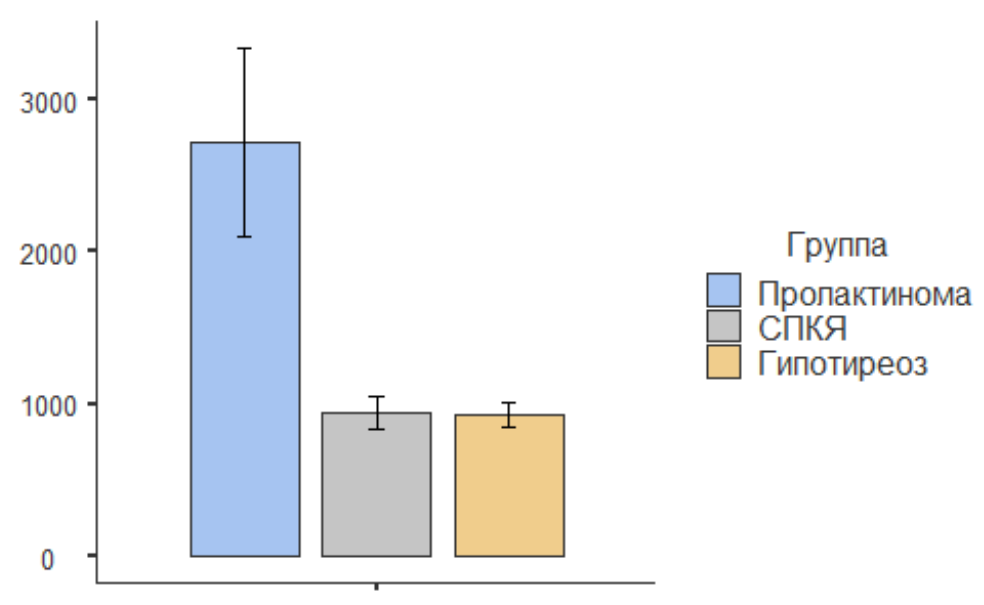

Пролактин мМЕ/Л (до)

Figure: 2. The level of total prolactin in the group of examined patients

\section{Determination of gonadotropic, thyroid hormones, testosterone and progesterone in blood serum in patients with hyperprolactinemia}

To obtain a comprehensive hormonal characterization of patients, we used the spectrum of hormonal studies. The serum hormone levels of the examined women are presented in Table 2.

When assessing the dependence of testosterone levels in women with hyperprolactinemia on its cause, significant differences were obtained between 2 (PCOS) and 1 (prolactinoma) (Kruskal - Wallis criterion, p1-2 <0.001) and between 2 (PCOS) and 3 (hypothyroidism) groups (p2-3<0.001), that is, testosterone levels in women with PCOS are much higher compared to women with prolactinoma and hypothyroidism.

Statistical analysis of the level of FSH, LH and progesterone in tumor and non-tumor hyperprolactinemia did not reveal significant differences, $p>0.05$.

Elevated FSH levels are observed in 3 women with prolactinoma, and in 6 patients with hypothyroidism. With PCOS, there is no increase in FSH levels. Elevated LH levels are observed in 3 women with prolactinoma, 3 with PCOS. 
Table 2. Levels of gonadotropic, thyroid hormones, testosterone and progesterone in the blood serum of the examined women (on day 21 menstrual cycle)

\begin{tabular}{|c|c|c|c|c|}
\hline & $\begin{array}{l}\text { Prolactinoma } \\
\quad(n=30)\end{array}$ & $\begin{array}{c}\text { PCOS } \\
(n=30)\end{array}$ & $\begin{array}{l}\text { Hypothyroidism } \\
(n=30)\end{array}$ & $\begin{array}{c}\mathrm{P}(\text { Kruskal - } \\
\text { Wallis criterion) }\end{array}$ \\
\hline $\mathrm{FSH} I U / \mathrm{L}$ & $4,7(1,9-6,92)$ & $3.6(2.4-6.6)$ & $2.36(2.2-6.88)$ & $\begin{array}{c}0.728 \\
P_{1}-2=1.0 \\
R 1-3=0,77 \\
P_{2}-3=0,77\end{array}$ \\
\hline $\mathrm{LH} I U / \mathrm{L}$ & $6(1.9-7.9)$ & $5.1(3.2-10.2)$ & $3.28(1.2-9.8)$ & $\begin{aligned} & 0.231 \\
& \mathrm{P}_{1}-2=0.82 \\
& \mathrm{R} 1-3=0,677 \\
& \mathrm{P} 2-3=0,163\end{aligned}$ \\
\hline LH/FSH & 1,276 & 1.417 & 1,389 & $\begin{array}{c}0.128 \\
P_{1}=0.91 \\
P_{2}=0.68 \\
P_{3}=0.175\end{array}$ \\
\hline Progesterone $\mathrm{ng} / \mathrm{ml}$ & $3.85(0.608-5.7)$ & $1.8(0.4-3.01)$ & $1.8(0.8-2.35)$ & $\begin{aligned} 0.277 \\
\mathrm{P} 1-2=0.499 \\
\mathrm{P} 1-3=0,256 \\
\mathrm{P}_{2}-3=0,955\end{aligned}$ \\
\hline Testosterone $\mathrm{ng} / \mathrm{ml}$ & $0.99 \pm 0.85$ & $2.2 \pm 1.04$ & $0.5 \pm 0.23$ & $\begin{array}{c}0.001 \\
P_{1}-2<0.001 \\
P_{1}-3=0.063 \\
P_{2}-3<0.001\end{array}$ \\
\hline $\mathrm{TSH} \mathrm{mlU} / \mathrm{ml}$ & $2.63 \pm 1.78$ & $2.03 \pm 1.0$ & $12.2 \pm 5.56$ & $\begin{array}{c}0.001 \\
\mathrm{P} 1-2=0.323 \\
\mathrm{P} 1-3<0.001 \\
\mathrm{P}_{2}-3<0.001\end{array}$ \\
\hline Free-T4 nmol/l & $14.7 \pm 3.12$ & $11.1 \pm 3.04$ & $4.2 \pm 3.16$ & $\begin{array}{c}0.001 \\
\mathrm{P}_{1-2}<0.001 \\
\mathrm{P} 1-3<0.001 \\
\mathrm{P}_{2}-3<0.001\end{array}$ \\
\hline
\end{tabular}

In patients with hypothyroidism, an excess of $\mathrm{LH}$ levels from the reference values is not observed. Low FSH levels are observed in 8 patients with prolactinoma, 7 with PCOS, and 6 with hypothyroidism. Low LH levels are noted in 1 patient with prolactinoma, 1 with PCOS, and 6 with hypothyroidism. According to the levels of LH and FSH, it can be concluded that with hyperprolactinemia syndrome, 
normogonadotropic hypogonadism develops mainly.

It was noted that in 9 women with prolactinoma, in 18 women with

PCOS and in 3 women with hypothyroidism, the ratio of $\mathrm{LH}$ to $\mathrm{FSH}$ exceeds the reference values ( $\mathrm{LH} / \mathrm{FSH}>1.5)$.

14 women with prolactinoma, 23 with PCOS, and 18 with hypothyroidism have low progesterone levels in the middle of the luteal phase of the menstrual cycle, which means chronic anovulation in women with hyperprolactinemia syndrome.

Increased testosterone levels are mainly found in patients with PCOS (observed in 17 patients). In 3 patients with prolactinoma, this indicator is higher than the reference values.

A low level of free T 4 is observed in 14 women with hypothyroidism (overt hypothyroidism).

\section{Study of the hypothalamic - pituitary - gonadal function in women with hyperprolactinemia syndrome}

Hyperprolactinemia is one of the most common causes of ovulation disorders, menstrual irregularities and infertility in women. Hyperprolactinemia is a heterogeneous group of neuroendocrine disorders from a clinical and pathogenetic point of view $[13,14,20]$.

To study structural changes in the ovaries and uterus, to assess ovulatory function and to diagnose gynecological diseases, tumors of the uterus, appendages, to identify abnormalities in the development of the uterus, the patients underwent ultrasound of the pelvic organs on days 14 and 16 of the menstrual cycle.
An ultrasound examination of the pelvic organs $(n=90)$ showed signs of sexual infantilism in 43 patients. These were mainly patients with tumor-induced hyperprolactinemia (prolactinoma). Twenty-one patients had signs of polycystic ovary (an increase in ovarian volume $>10 \mathrm{ml}$, the number of antral follicles in one cut $\geq 12$, with dimensions of 2-9 mm, thickening of the ovarian stroma) (PCOS-21), 16 had signs of a multifollicular structure of the ovaries (prolactinoma - 5, PCOS - 8, hypothyroidism - 3). Among patients with hyperprolactinemia of non-neoplastic genesis, 3 had small uterine fibroids, 2 had signs of adenomyosis, 4 patients had signs of chronic inflammatory disease of the pelvic organs without exacerbation, and one patient had a dermoid cyst of the right ovary. LH and FSH levels are presented in Table 2.

Evaluating the clinical signs in patients with tumor-induced hyperprolactinemia, depending on the level of total prolactin, it was shown that in women with amenorrhea the prolactin level was significantly higher (Kruskal-Wallis criterion $p=0.01$ ). Thus, evaluating the clinical signs, the levels of prolactin, FSH, LH and progesterone, as well as on the basis of ultrasound of the pelvic organs, it can be concluded that in hyperprolactinemia of tumor genesis, the severity of menstrual irregularities depends on the level of prolactin (with the exception of the phenomenon of macroprolactinemia).

\section{MRI of the hypothalamic - pituitary region in the diagnosis of prolactinomas}

MRI of the hypothalamic - pituitary region was performed in all patients with prolactinoma to visualize and determine the size of the tumor, the state of chiasm, and to exclude other changes in the hypothalamic-pituitary region. 
The characteristics of the MRI results are presented in Table 3.

Table 3. Results of MRI in patients with prolactinoma

\begin{tabular}{|c|c|c|}
\hline $\begin{array}{c}\text { Tumor volume, } \\
\mathrm{mm}^{3}\end{array}$ & $\begin{array}{c}\text { Microprolactinomas } \\
\mathrm{N}=25(\mathrm{p}=0.23)\end{array}$ & $\begin{array}{c}\text { Macroprolactinomas } \\
\mathrm{N}=5(\mathrm{p}=0.001)\end{array}$ \\
\hline Median, $\mathrm{mm}^{3}$ & 42.39 & 1778.86 \\
\hline $\begin{array}{c}\text { Lower quartile, } \\
\mathrm{mm}^{3}\end{array}$ & 22.30 & 900.34 \\
\hline $\begin{array}{c}\text { Upper quartile, } \\
\mathrm{mm}^{3}\end{array}$ & 118.90 & 4056.78 \\
\hline
\end{tabular}

Thus, MRI study, a highly informative noninvasive method of radiological diagnosis, revealed pituitary microadenomas in 25 cases (83.3\%), macroadenomas in $5(16.7 \%)$ cases. With the aid of MRI of the hypothalamic pituitary region, we identified the pituitary gland and the tumor size, tumor growth pattern, state chiasm and other brain structures. Given the relatively high cost of MRI studies, this method should be carried out strictly according to indications in the presence of clinical signs, as well as for dynamic monitoring of patients with prolactinoma during treatment with dopamine agonists [21].

\section{CONCLUSIONS}

1. When assessing the dependence of the level of total prolactin on the cause of hyperprolactinemia, between tumor hyperprolactinemia (prolactinoma) and non-tumor genesis (PCOS, hypothyroidism), significant differences were obtained (Kruskal -
Wallis criterion, p 1-2 <0.001, p $13<0.001)$.

2. Testosterone levels in women with PCOS are much higher compared to women with prolactinoma and hypothyroidism.

3. Evaluating clinical signs, levels of prolactin, FSH, LH and progesterone, as well as on the basis of ultrasound of the pelvic organs, it can be concluded that with hyperprolactinemia of tumor genesis, the severity of menstrual irregularities depends on the level of prolactin (with the exception of the phenomenon of macroprolactinemia).

4. MRI examination of the hypothalamicpituitary region, a highly informative non-invasive method of radiological diagnostics, revealed pituitary microadenomas in 25 cases $(83.3 \%)$, macroadenomas in $5(16.7 \%)$ cases. 


\section{REFERENCES}

1. Vaks V.V. Hyperprolactinemia: causes, clinical picture, diagnosis and treatment. // Consilium-Medicum. 2001 - Volume 3. No. 11.

2. Vorotnikova S.Yu. Diagnostics and treatment of hyperprolactinemia: clinical guidelines of the International Endocrinological Society / Translation and comments by S.Yu. Vorotnikova, E.A. Pigarova, LK Dzeranova // Obesity and Metabolism. - 2011. - No. 2. - P. 79-94.

3. Vorotnikov, S.Yu. Diagnostics and treatment of hyperprolactinemia: clinical guidelines of the International Endocrinological Society / Translation and comments by S.Yu. Vorotnikova, E.A. Pigarova , L.K. Dzeranova // Obesity and metabolism. - 2011. - No. 2. - P. 79-94.

4. Hyperprolactinemia in women and men: a guide for doctors / GA Melnichenko [et al.]. - M., 2007.-- 33 p.

5. Hyperprolactinemia. Modern approaches and old problems / I.I. Dedov [and others] // Bulletin of reproductive health. - 2009. No. 2 (June). - S. 2-8.

6. Dzeranova L.K. Features of diagnosis and treatment of hyperprolactinemic syndrome / L.K. Dzeranova , I.I. Barnmina II Effective pharmacotherapy in endocrinology. - 2009. - No. 1. - S. 2-7.

7. Dzeranova LK, Syndrome of hyperprolactinemia in women and men: clinical picture, diagnosis, treatment. Dissertation. Abstract.

8. Diagnostics and therapy of moderate forms of hyperprolactinemia / I.A. Ilovaiskaya [and others] // Obstetrics and gynecology. - 2000. - number 4. - C. 19-22.

9. Mohort T.V., Safina M.R. Hyperprolactinemia syndrome: modern approaches to diagnosis and treatment. Reproductive health. Eastern Europe 2012. No. 4 (22) 130-141.

10. Casanueva FF, Molitch ME, Schlechte JA, Abs R, Bonert V, Bronstein MD, Brue T, Cappabianca $P$, Colao A, Fahlbusch R, Scalon M, Sobrinho LG, Wass JA, Giustina A 2006 Guidelines of the Pituitary Society for the diagnosis and management of prolactinomas. Clin Endocrinol (Oxf ) 65: 265-273.

11. Do the limits of serum prolactin in disconnection hyperprolactinaemia need redefinition? A study of 226 patients with histologically verified nonfunctioning pituitary macroadenoma / N. Karavitaki [et al.] // Clin. Endocrinol. (Oxf .). - 2006. - Vol. 65. - P. 524-529.

12. Donadio F., Barbieri A., Angioni R., Mantovani G., Beck- Peccoz P., Spada A., Lania AG 2007 Patients with macroprolactinemia: clinical and radiological features. Eur J Clin Invest 37: 552-557.

13. Hattori N. 2003 Macroprolactinemia: a new cause of hyperprolactinemia . J Pharmacol Sci 92: 171-177.

14. Kars M., Souverein PC, Herings RM, Romijn JA, Vandenbrouche JP, de Boer A., Dekkers OM 2009 Estimated age- and sexspecific incidence and prevalence of dopamine agonist-treated hyperprolactinemia. J Clin Endocrinol Metab 94: 2729-2734.

15. Long-term follow-up of patients with hyperprolactinaemia / WJ Jeffcoate // Clin. Endocrinol. (Oxf .). - 1996. - Vol. 45, No. 3. P. 299-303.

16. Mah PM, Webster J. Hyperprolactinemia: Etiology, Diagnosis, and Management. SeminReprodMed 2002; 20: 4: 365-374. 
17. Mancini T, Casanueva FF, Giustina A 2008 Hyperprolactinemia and prolactinomas. Endocrinol Metab Clin North Am: 37: 67-

99.

18. Shlomo Melmed, Felipe F. Casanueva, Andrew R. Hoffman, David L. Kleinberg, Victor M. Montori , Janet A. Schlechte, and John AH Wass. Diagnosis and Treatment of Hyperprolactinemia: An Endocrine Society Clinical Practice Guideline. Endocrine Society 2011.273-288.

19. Thompson JA, Gray CE, Teasdale GM. Relapse of hyperprolactinemia after transsphenoidal surgery for microprolactinoma: lessons from longterm follow-up. Neurosurgery 2002; 50: 36-9. [ PubMed]

20. Thorner MO, Bronstein M. Pituitary disease and neuroendocrinology. Ed. A. Grossman. Prolactin. Disorders 2011; 6.

21. Vilar $L$, Freitas MC, Naves LA, Casulari LA, Azevedo M, Barros Al, Faria M, Lima JG, Cruz TP, Mota A, Ramos A, Violante A, Lamounier Filho A, Gadelha MR, Czepielewski MA, Glezer A, Bronstein MD 2008 Diagnosis and management of hyperprolactinemia: results of a Brazilian. 